\title{
Inhibition of STAT3 reverses drug resistance acquired in temozolomide-resistant human glioma cells
}

\author{
EUN-SANG LEE ${ }^{1 *}, \mathrm{KYUNG} \mathrm{KON} \mathrm{KO}^{1 *}$, YOUNG AE JOE ${ }^{1}, \mathrm{SEOK}-G U_{\mathrm{KANG}}^{2}$ and YONG-KIL HONG ${ }^{1,2}$ \\ ${ }^{1}$ Cancer Research Institute and ${ }^{2}$ Department of Neurosurgery, College of Medicine, \\ The Catholic University of Korea, Seoul 137-701, Korea
}

Received May 12, 2010; Accepted October 12, 2010

DOI: $10.3892 / \mathrm{ol} .2010 .210$

\begin{abstract}
The alkylating agent temozolomide (TMZ) is an effective drug used for the treatment of malignant gliomas. However, tumor relapse combined with the development of drug resistance remains a significant problem. To clarify the mechanism of the resistance of glioma cells to TMZ chemotherapy, TMZ-resistant glioma cell lines (TR cells) were generated using U373 and U251 human glioma cells, and TMZ-resistance was confirmed via viability and apoptosis assays. The TMZ-resistance of TR cells was not associated with the TMZ-resistance molecule $\mathrm{O}^{6}$-methylguanine-DNAmethyltransferase. Notably, the expression level of signal transducers and activators of transcription 3 (STAT3) and serine 727-phosphorylated STAT3 (pSTAT3-Ser727) was highly increased in TR cells, while that of 705-phosphorylated STAT3 (pSTAT3-Tyr705) was decreased. The inhibition of STAT3 expression by small interfering RNA enhanced TR cell TMZ sensitivity. These results suggest that STAT3 contributes to TMZ-resistance in gliomas and is a potential target for the reversal of TMZ-resistance in patients with a recurrent glioma.
\end{abstract}

\section{Introduction}

Malignant glioma is a highly aggressive tumor with a poor prognosis despite advances in surgery, radiotherapy and chemotherapy (1). Temozolomide (TMZ), a new generation of oral alkylating agents that induces apoptosis by methylation of the $\mathrm{O}^{6}$-position of guanine, is considered to be one of the most effective chemotherapeutic agents against malignant glioma (2). Its clinical efficacy is limited in most cases by recurrence or the development of resistance (3). Various approaches have been investigated to overcome the resistance and improve

Correspondence to: Dr Yong-Kil Hong, Department of Neurosurgery, Seoul St. Mary's Hospital, The Catholic University of Korea, Banpo-dong 505, Seocho-gu, Seoul 137-701, Korea

E-mail: hongyk@catholic.ac.kr

*Contributed equally

Key words: temozolomide, drug resistance, STAT3, glioma the efficacy of TMZ, including dose-intense scheduling (4), combined therapy with radiotherapy (2), angiogenesis inhibition (5), immune modulators (6) and other chemotherapies (7). The mechanism of TMZ resistance remains unclear. An understanding of the resistance mechanism is crucial if the clinical efficacy of TMZ is to be improved.

Signal transducers and activators of transcription 3 (STAT3) is a cytoplasmic transcription factor that is phosphorylated by various protein tyrosine kinases including Janus kinases and $\mathrm{Src}$ in response to the activation of cytokines and growth factors $(8,9)$. Phosphorylation at tyrosine 705 (pSTAT3-Tyr705) induces its dimerization, nuclear translocation and DNA binding (10), and phosphorylation at Serine 727 (pSTAT3-Ser727) via the MAPK or mTOR pathway appears to regulate transcriptional activation $(8,11)$. STAT3 plays a key role in cell proliferation, apoptosis and differentiation (12). STAT3 is transiently phosphorylated in normal cells, whereas it is constitutively activated in the majority of solid tumor cells (13). Aberrant activation of this protein affects tumorigenesis and interferes with tumor chemotherapy (14). The results of numerous studies are in agreement with the suggestion that the STAT3 oncogenic pathway is related to drug resistance and the prevention of cell death through intrinsic and/or extrinsic apoptotic pathways. Constitutively-activated STAT3 upregulates the expression of prosurvival proteins, including Bcl-xL and Mcl-1, which prevent cytochrome c release and inhibit mitochondria-dependent cell death (15). In addition, STAT3 has been reported to exhibits resistance to Fas-mediated apoptosis in multiple myeloma (16). Activated STAT3 is overexpressed in the majority of paclitaxel-resistant ovarian cancer cells, and therefore the inhibition of STAT3 activation results in a significantly decreased paclitaxel resistance and enhanced apoptosis (17). Drug-resistant recurrent tumors have increased STAT3 phosphorylation as compared with matched primary tumors.

This study was therefore designed to investigate the relationship between STAT3 and TMZ-resistance in gliomas.

\section{Materials and methods}

Cell lines and culture conditions. Human glioma cell lines U373, U251 and T98G were purchased from the American Tissue Culture Collection (Rockville, MD, USA). To develop TR cells, U373, U251 and T98G cell lines were initially 
cultured in the presence of $12.5 \mu \mathrm{M}$ of TMZ. The concentration was then increased by 2 -fold for every two passages until it reached $800 \mu \mathrm{M}$ (maximum concentration). The cancer cell lines were cultured in Dulbecco's Modified Eagle Medium (DMEM; WelGene, Dae-Ku, Korea) supplemented with $10 \%$ fetal bovine serum (FBS; WelGene) and $50 \mu \mathrm{g} / \mathrm{ml}$ gentamicin (Gibco, Grand Island, NY, USA).

Drugs. Stock solutions of TMZ (Schering-Plough, Kenilworth, NJ, USA) and Celecoxib (Pfizer, New York, NY, USA) were prepared by dissolving the drug in dimethyl sulfoxide (SigmaAldrich, St. Louis, MO, USA). Methotrexate (Sigma-Aldrich) was prepared by dissolving the drug in $10 \mathrm{mM}$ sodium chloride. 1,3-Bis(2-chloroethyl)-1-nitrosourea (BCNU; Bristol Myers Squibb, Princeton, NJ, USA) was reconstituted in sterile water.

Cell viability assay. Cell viability was assessed using the Cell Counting Kit-8 assay (CCK-8; Dojindo, Japan). Briefly, 500 cells (U373, U251, TR-U373 and TR-U251) were plated in each well of a 96-well plate. After $24 \mathrm{~h}$, the cells were treated with various concentrations of drugs including TMZ (20 mg/ $\mathrm{ml}), \mathrm{BCNU}(3.3 \mathrm{mg} / \mathrm{ml}), \operatorname{MTX}(1.5 \mu \mathrm{g} / \mathrm{ml})$ and Celecoxib $(100 \mathrm{mg} / \mathrm{ml})$ for 5 days and the dose-response was analyzed. The cells were incubated with CCK-8 solution for $2 \mathrm{~h}$ and the absorbance was measured at $450 \mathrm{~nm}$ using an ELISA reader (Molecular Devices, Sunnyvale, CA, USA). The percentage of cell viability was calculated relative to the untreated cells as the control.

Apoptosis analysis by flow cytometry. An Annexin V-PE apoptosis kit (BD Biosciences, Franklin Lakes, NJ, USA) was used according to the manufacturer's instructions. Briefly, U373, U251, TR-U373 and TR-U251 cells were treated with TMZ for 5 days prior to being harvested and washed with cold PBS. The washed cells were resuspended in Annexin V binding buffer followed by staining with Annexin V-PE together with 7-AAD. The stained cells were then analyzed by FACS (Becton-Dickinson, Franklin Lakes, NJ, USA) and the results were analyzed using Cell Quest software (BectonDickinson).

Western blot analysis. Cells were serum-starved for $24 \mathrm{~h}$ and then pre-stimulated with $20 \mathrm{ng} / \mathrm{ml} \mathrm{IL-6}$ for $20 \mathrm{~min}$ immediately prior to harvesting. Protein lysates were subjected to $10 \%$ SDS-PAGE and the resolved proteins were transferred electrophoretically onto nitrocellulose membranes (Pall, Pensacola, FL, USA). Membranes were blocked with 5\% skim milk and incubated with specific antibodies; anti- ${ }^{6}$ methylguanine-DNA-methyltransferase (MGMT; Chemicon, Temecula, CA, USA), anti-STAT3 (Santa Cruz Biotechnology, Santa Cruz, CA, USA), anti-phospho-STAT3 Y705 (Cell Signaling, Beverly, MA, USA), anti-phospho-STAT3 S727 (Cell Signaling) and anti- $\beta$-actin (Sigma-Aldrich). The blots were developed using a chemiluminescence detection system (Amersham Biosciences, Piscataway, NJ, USA).

STAT3 small interfering RNA transfection. U373, U251, TR-U373 and TR-U251 cells were transfected with STAT3 small interfering RNA (siRNA; $80 \mathrm{nM}$ ) or control siRNA
(80 nM) using Lipofectamine RNAiMAX (Invitrogen, Carlsbad, CA, USA). Briefly, one day prior to transfection, the cells $\left(1 \times 10^{3}\right)$ were either plated in 96 -well plates to determine cell viability or on $60 \mathrm{~mm}$-diameter dishes $\left(1 \times 10^{5}\right)$ for Western blot analysis with 10\% FBS DMEM (WelGene) without antibiotics. siRNA-lipofectamine complex mixture in serum-free Opti-MEM (Gibco, Grand Island, NY, USA) was prepared according to the manufacturer's instructions. The medium was replaced with DMEM containing 10\% FBS $4 \mathrm{~h}$ after transfection. After $48 \mathrm{~h}$, the protein extracts were prepared for Western blot analysis and the cell viability was measured following additional treatment with TMZ for $72 \mathrm{~h}$.

\section{Results}

Generation of temozolomide-resistant glioma cell lines. TR-U373 and TR-U251 cells were generated from the parental cell lines by treatment with 2-fold increasing TMZ concentrations (12.5-800 $\mu \mathrm{M}$ TMZ) every two passages over a period of 2 months. TMZ-resistance of the established TR cells was evaluated by viability analysis using a CCK-8 assay. TR-U373 and TR-U251 were 16- and 10-fold, respectively, more resistant to $\mathrm{TMZ}$ as compared with the parental cells $\left(\mathrm{IC}_{50}, 800 \mu \mathrm{M}\right.$ vs. $50 \mu \mathrm{M}$ and $1,000 \mu \mathrm{M}$ vs. $100 \mu \mathrm{M}$, respectively; Fig. 1A). TMZ-resistance of the TR cells was maintained with the absence of drugs for almost 2 months (data not shown). In addition, the TR cells exhibited no resistance to various anti-cancer drugs including MTX, Celecoxib, and BCNU (Fig. 1B).

Resistance to temozolomide-induced apoptosis in TR-U373 and TR-U251 cells. Following exposure to $200 \mu \mathrm{M}$ TMZ for 5 days, the cellular morphology of the TR cells was stably altered as compared with the parental cells (Fig. 2A). Since TMZ causes cell death through apoptosis (18), TMZ-induced apoptosis was compared between parental and TR cells. Apoptosis induced by $200 \mu \mathrm{M}$ TMZ was evaluated with Annexin V/7-AAD staining by FACS. Following exposure to TMZ, the early apoptotic Annexin $\mathrm{V}^{+} / 7-\mathrm{AAD}^{-}$cells were noted to have decreased in TR-U373 and TR-U251 cells compared to those of the parental cells, from 37 to $4 \%$ and 49 to $11 \%$, respectively. However, the incidence of necrotic Annexin $\mathrm{V}^{-} / 7-\mathrm{AAD}^{+}$or late apoptotic Annexin $\mathrm{V}^{+} / 7-\mathrm{AAD}^{+}$ cells showed no significant differences (Fig. 2B). The results showed that TR cells are resistant to TMZ-induced apoptosis.

Temozolomide-resistance and MGMT expression. The MGMT DNA repair protein contributes to drug resistance through the demethylation of methyl adducts formed by TMZ (19). To investigate whether TMZ-resistance occurring in TR cells is due to the expression of MGMT, the expression of MGMT in TR cells derived from MGMT non-expressing U373 and U251 cells was assessed. Western blot analysis detected MGMT expression in T98G and TR-T98G cells, but not in U373, U251, TR-U373 and TR-U251 cells (Fig. 3A). Accordingly, TR cells showed a strong resistance to TMZ, although MGMT expression was not detected. The results suggest that TMZ resistance acquired in TR cells is related to other molecules or factors. 
A
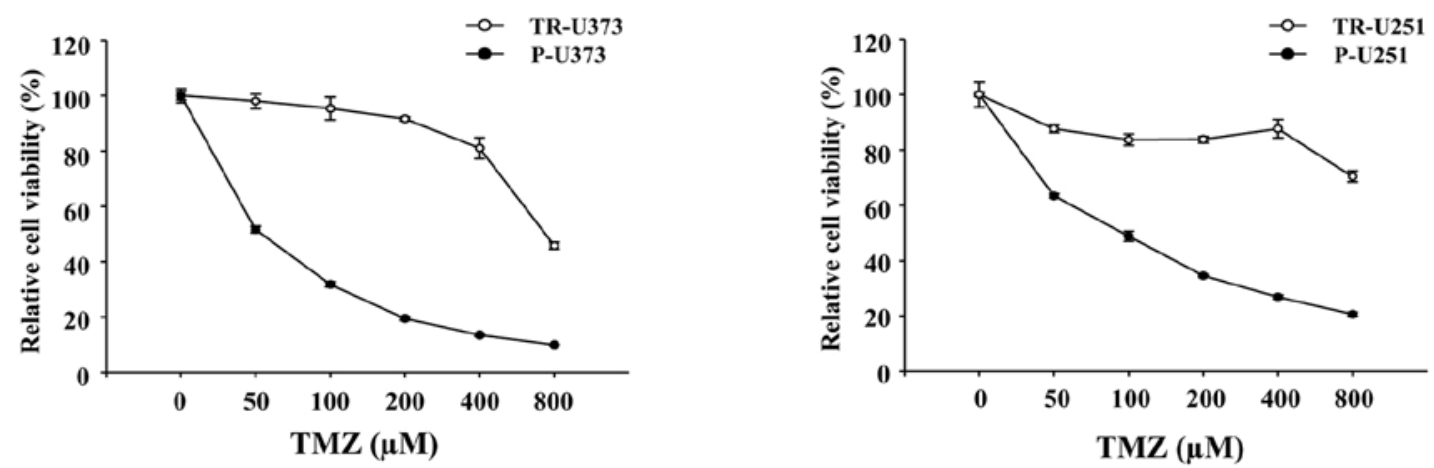

B
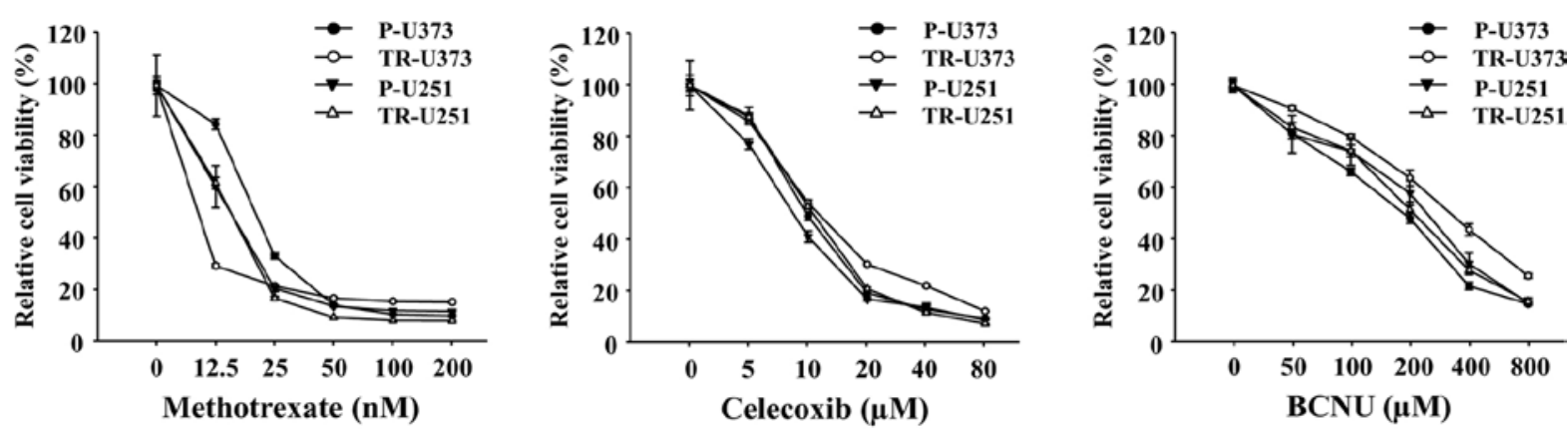

Figure 1. Cell viability in the parental and TR cells. (A) The parental (U373 and U251) and TMZ-resistant (TR-U373 and TR-U251) cells were seeded in 96-well plates and treated with TMZ for 5 days prior to the determination of cell viability. Data are the mean \pm SD of five independent experiments. (B) Effect of other anti-cancer drugs on cell viability in TR cells. The parental and TR cells were exposed to MTX, Celecoxib or BCNU for 5 days prior to the determination of cell viability. Data are the mean \pm SD of three independent experiments. P, parental cells; TR, TMZ-resistant cells.

A

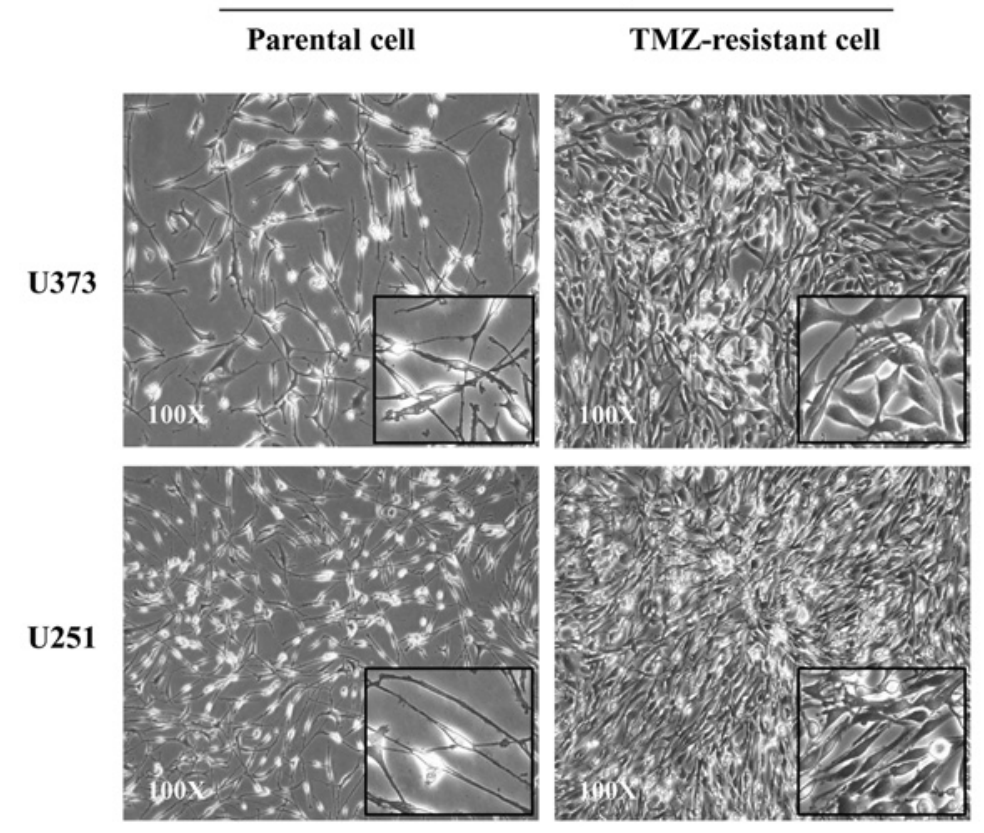

B

$\frac{\text { Treatment with } 200 \mu \mathrm{M} \text { TMZ }}{\text { Parental cell } \quad \text { TMZ-resistant cell }}$
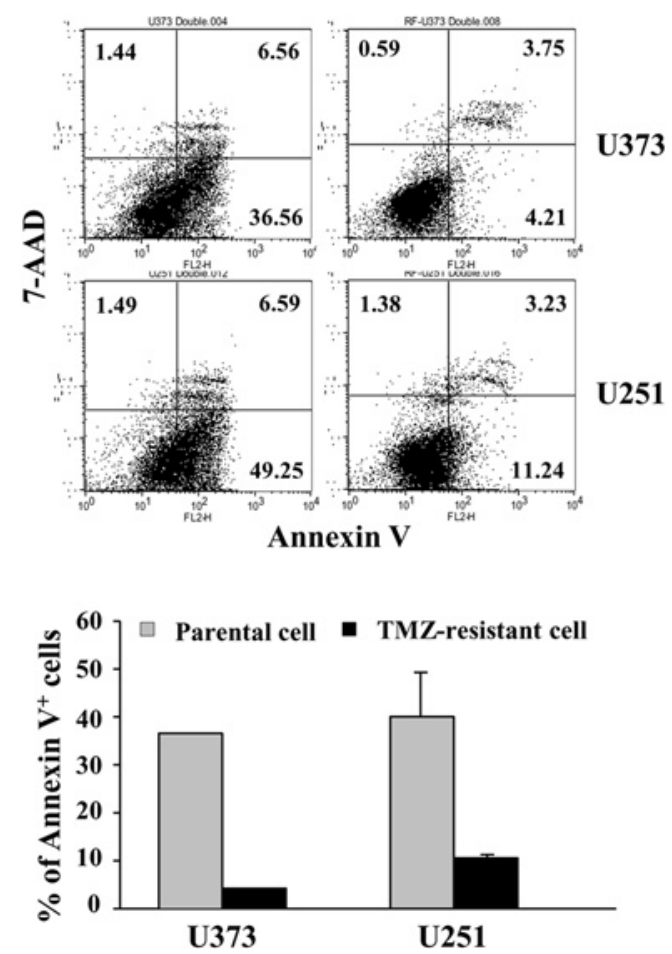

Figure 2. TMZ-induced apoptosis in the parental and TR cells. (A) Phase contrast images showing the morphology of the parental and TR cells following 5 days of exposure to $200 \mu \mathrm{M}$ TMZ. These cells exhibited a stable morphology and survival compared with the parental cells. (B) The parental and TR cells were treated with $200 \mu \mathrm{M}$ TMZ for 5 days and stained with PE-conjugated Annexin V and 7-AAD. The apoptotic cells were measured by FACS analysis. The results are shown as the percentage of apoptotic cells. Lower right quadrant, Annexin $\mathrm{V}^{+} / 7-\mathrm{AAD}^{-}$, early apoptotic cells; upper right quadrant, Annexin $\mathrm{V}^{+} / 7-\mathrm{AAD}^{+}$, late apoptotic cells. 
$\mathbf{A}$

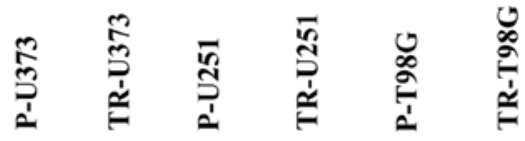

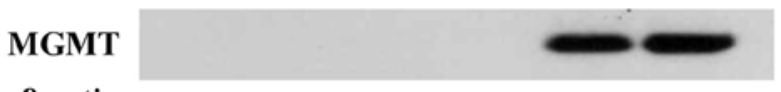

$\beta$-actin

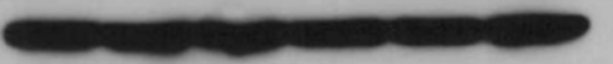

B
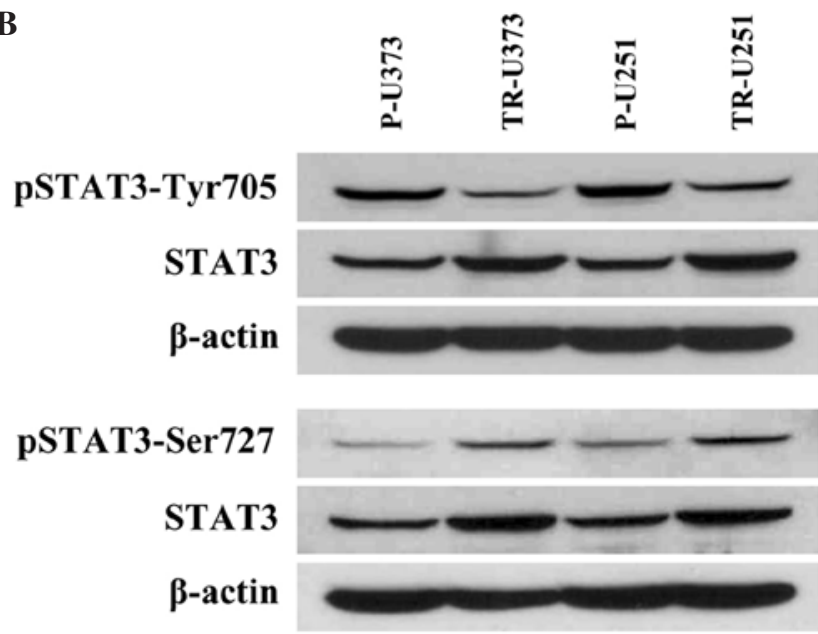

C

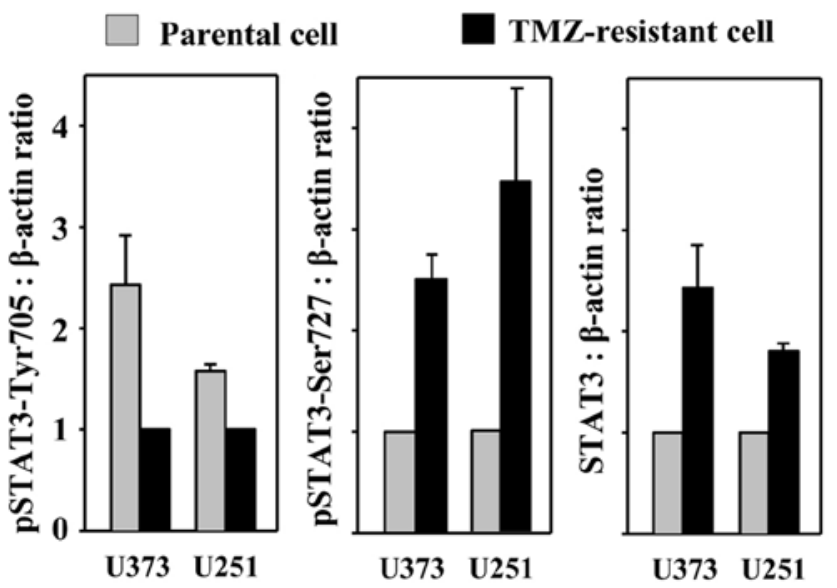

Figure 3. The expression of MGMT and STAT3 in parental and TR cells. (A) MGMT expression was examined in the parental and TR cells of U373, U251 and T98G by Western blot analysis. MGMT was expressed only in T98G cells (MGMT positive control) of the parental and TR cells. $\beta$-actin was used as a loading control. (B) The parental and TR cells were stimulated with IL-6 (20 ng/ml, $20 \mathrm{~min})$ prior to harvesting. Total cell lysates were subjected to Western blot analysis to detect the expression levels of STAT3, pSTAT3-Tyr705 and pSTAT3-Ser727 using the specific antibodies as indicated, with $\beta$-actin as the loading control. Representative images are shown from one of four independent experiments, which produced similar results. (C) Densitometric analysis assessing the relative protein expression levels in three independent experiments. The results are the mean \pm SD P, parental cells; TR, TMZ-resistant cells.

Increased activation and expression of STAT3 in temozolomideresistant glioma cell lines. STAT3 is highly expressed and activated in paclitaxel-resistant ovarian cancer cells (17). To determine whether the expression and activation of STAT3 increased in TR cells, the expression level of STAT3 and phosphorylated STAT3 were investigated in the parental and TR cells by Western blot analysis. The levels of STAT3 and pSTAT-Ser727 were increased in the TR cells as compared with the parental cells. On the other hand, the level of pSTAT3Tyr705 was decreased (Fig. 3B). TR-U373 and TR-U251 cells exhibited an 2.4 - and 1.8-fold increased level of STAT3, respectively, and an 2.3- and 2.9-fold increased level of pSTAT3-Ser727, respectively, whereas the level of pSTAT3Tyr705 was decreased by $2.4-$ and 1.6 -fold, respectively (Fig. 3C).

Reversal of temozolomide-resistance using small interfering RNA in TR-U251 cells. Based on the observation that the level of STAT3 is elevated in TR cells, an experiment was performed to assess whether the inhibition of STAT3 expression would increase TMZ sensitivity in TR cells. First, to evaluate the knockdown efficiency of siRNA, TR-U251 cells were treated with various concentrations of STAT3 siRNA. STAT3 expression was dose-dependently inhibited by STAT3 siRNA and its efficacy was maximized at $80 \mathrm{nM}$ (Fig. 4A, left panel). In addition, to determine the optimal incubation time after post-transfection based on the experimental conditions, the expression of STAT3 was determined at various time points following transfection by Western blotting. Knockdown of STAT3 expression by siRNA was optimized at $96 \mathrm{~h}$ and maintained for $96 \mathrm{~h}$ (Fig. 4A). Based on these conditions, the effects of STAT3 knockdown and TMZ treatment were determined. Both parental-U251 (P-U251) and TR-U251 cells were transfected with STAT3 siRNA for $48 \mathrm{~h}$ and then incubated in the absence or presence $(200 \mu \mathrm{M})$ of TMZ for a further $72 \mathrm{~h}$. Co-treatment with STAT3 siRNA and TMZ resulted in synergistic decreases in cell viability as compared to each treatment alone (Fig. 4B). Notably, TMZ-resistant TR-U251 cells showed a relatively comparable effect on combination treatment compared to TMZ-sensitive P-U251 cells. This result was consistent with the association of STAT3 with TMZ-resistance, thereby indicating that the inhibition of STAT3 is able to reverse TMZ-resistance in TR cells.

\section{Discussion}

TMZ is considered to be the most effective drug in the treatment of glioma. TMZ is also regarded as the standard chemotherapeutic drug in combination with surgery and radiotherapy (2). However, its efficacy is often limited by tumor recurrence due to the development of resistance to TMZ (3). Since the mechanism behind TMZ-resistance development is unclear, the present study was undertaken in order to generate TR cells to determine the molecular mechanism of TMZ-resistance development.

TR cells were generated by exposure to a gradually increasing TMZ concentration. Analysis of TMZ-resistance in TR cells indicated that the resistant cells exhibit a strong resistance to TMZ and have a significantly diminished progression of apoptosis compared with the parental cell lines (Figs. 1 and 2). Such resistance was stable upon the removal of TMZ and was maintained for a considerable period of time. Earlier studies reported that cancer cells exposed to an anticancer drug show resistance to diverse anticancer drugs that are structurally and functionally different from the primary anticancer 


\section{siSTAT3 concentration (nM)}

$\begin{array}{llllll}0 & 5 & 10 & 40 & 80 & h\end{array}$

STAT3

$\beta$-actin

\section{Post-transfection incubation time}

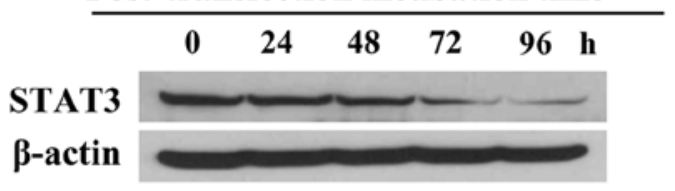

B

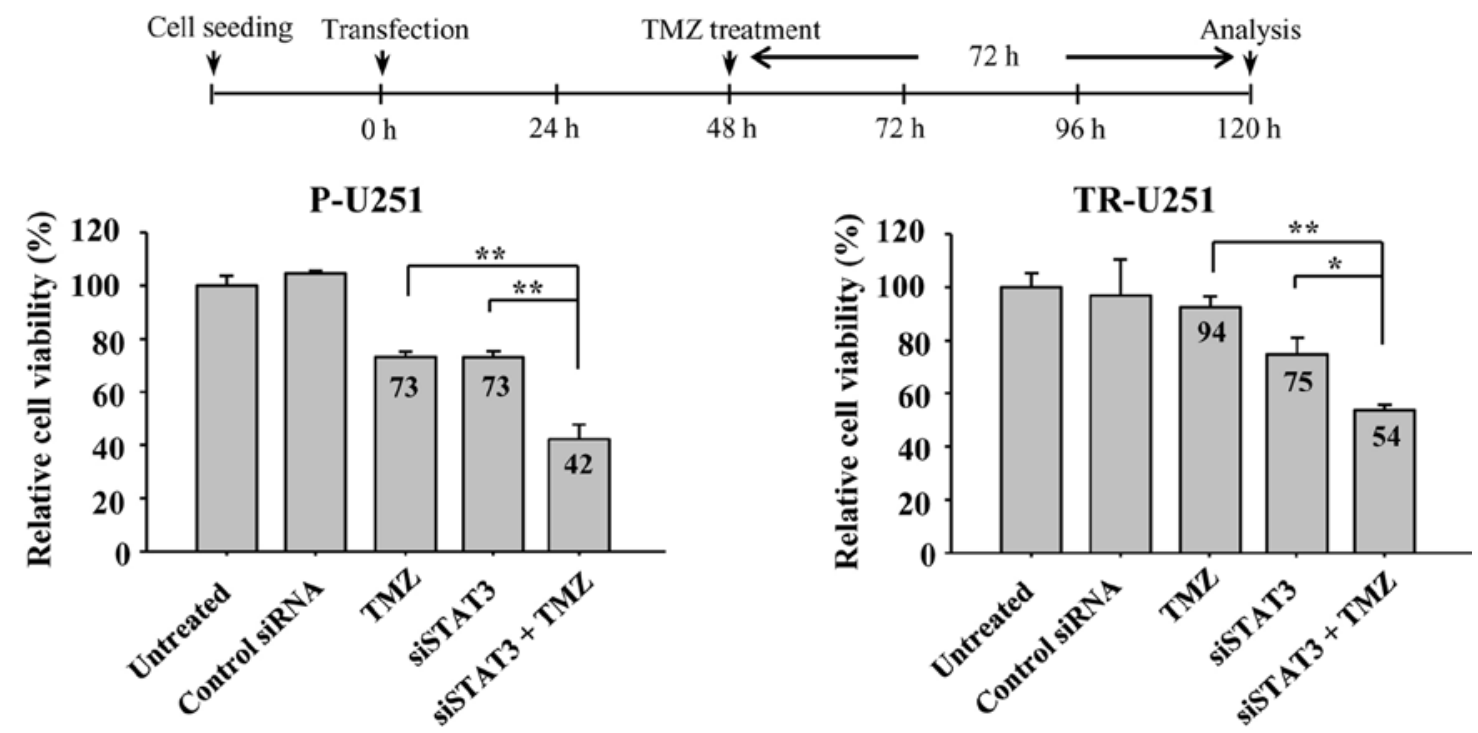

Figure 4. The effect of siRNA-induced inhibition of STAT3 expression in TR-U251 cells. (A) Knockdown of STAT3 expression by siRNA was successfully performed and regulated by siRNA concentration and post-transfection incubation in a time-dependent manner. TR-U251 cells were treated at the indicated concentrations of STAT3 siRNA for $48 \mathrm{~h}$ (left panel). Cells treated with STAT3 siRNA $(80 \mathrm{nM})$ were incubated for the indicated times (right panel). The expression of STAT3 was detected by Western blotting and $\beta$-actin was used as a loading control. (B) A schematic representation of the experimental protocol is shown in the upper panel. The cells were seeded $24 \mathrm{~h}$ prior to STAT3 siRNA transfection $(80 \mathrm{nM})$ and TMZ with $200 \mathrm{nM}$ was treated for $72 \mathrm{~h}$ following $48 \mathrm{~h}$ of siRNA transfection. The inhibition of STAT3 expression reversed TMZ-resistance in TR-U251 cells. Cell viability was measured using a CCK-8 assay. ${ }^{*}, \mathrm{P}<0.01 ;{ }^{* *}, \mathrm{P}<0.001$.

drug $(20,21)$. In contrast, the TR cells used showed resistance to TMZ but not to other tested drugs, when compared with the parental cells (Fig. 1). These observations are in agreement with the previously reported result that the viability of BCNUresistant leukemia cells is subject to inhibition by TMZ (22). Thus, it can be considered that TR cells that develop resistance solely to TMZ are a more useful model for the investigation of TMZ-resistance in glioma cells.

TMZ is converted to monomethyl triazeno imidazole carboxamide in the body, which in turn induces apoptosis in glioma cells by methylating the $\mathrm{O}^{6}$-position of guanine. However, this effect of TMZ is obstructed by MGMT which demethylates the methyl adduct formed by TMZ (19). MGMT, which is expressed in a subgroup of gliomas, plays a key role in cellular resistance to alkylating agents including TMZ, BCNU and cyclophosphamide (23). A real-time PCR analysis revealed a strong expression of the gene encoding MGMT in BCNU-resistant leukemia cells as compared with no detectable MGMT gene expression in parental leukemia cells (22). However, in this study, MGMT was not expressed in either parental or U373 and U251 TR cells. Moreover, the expression of MGMT in T98G cells did not differ between the parental and TR cells (Fig. 3A). These observations provide evidence that other molecules or factors affect the resistance to TMZ.

Previous studies reported that STAT3 is associated with drug resistance as well as tumor cell proliferation and apoptosis. STAT3-dependent overexpression of $\mathrm{Bcl}-2$ confers a survival advantage to breast cancer cells and contributes to their chemoresistance (24). The expression and activation of STAT3 is significantly increased in paclitaxel-resistant ovarian cancer cells and tissues, and the inhibition of STAT3 reduces paclitaxel resistance $(17,25)$. Similar findings are reported in DOX-resistant human cancer cells including breast and liver cancer and neuroblastoma $(26,27)$. However, the role of STAT3 in the acquisition of TMZ-resistance in glioma has yet to be determined. Therefore, the present study investigated the relationship between STAT3 and TMZ-resistance in glioma cells. STAT3 and pSTAT3-Ser727 were overexpressed in TR cells as compared with the parental cells, whereas the expression of pSTAT3-Tyr705 was decreased in TR cells (Fig. 3B).

With regard to STAT3 activity, it was reported that as STAT3-Tyr705 is activated, drug resistance is increased accordingly $(17,27)$. STAT3 is activated by phosphorylation on tyrosine residue 705 in response to cytokines such as IL-6 to form a homodimer (28). This activation transfers the expression of genes such as Bcl-2, survivin and cyclin D1. STAT3 may be correlated to drug resistance through tyrosine phosphorylation (24-26). In the present study, however, TMZ-resistance was increased in a viability assay while pSTAT-Tyr705 was decreased in TR cells (Fig. 1A). When the cells were treated with IL-6 to stimulate STAT3, Western blot analysis showed a decreased expression of pSTAT3-Tyr705 in TR cells compared to the parental cells. It is conceivable that the phosphorylation of STAT3 on tyrosine residue 705 in TR cells may not be 
stimulated by IL-6, and pSTAT3-Tyr705 may not be directly associated with TMZ-resistance acquired in TR cells.

STAT3 has another phosphorylation site on the serine 727 residue phosphorylated in response to IL-6 and other factors $(8,29)$. Serine phosphorylation is mediated either directly or indirectly by a signal transduction cascade involving MAPK, mTOR and PKCS (11,30,31), and it appears to regulate transactivation with tyrosine phosphorylation. Recent studies have proposed that serine 727 phosphorylation is sufficient to activate STAT3 without tyrosine 705 phosphorylation, as is noted, for example, in the activation of STAT3 in macrophages (32) or chronic lymphocytic leukemia cells (33). Moreover, the activation of STAT3 through serine phosphorylation promotes prostate tumorigenesis independent of tyrosine phosphorylation (34). These reports are in agreement with our result in that the expression of pSTAT3-Ser727 was increased in TR cells as compared with the parental cells. In addition, phosphorylation of STAT3 on the serine 727 residue is enhanced during mitosis and this correlates with a reduction of tyrosine 705 phosphorylation $(35,36)$. Therefore, serine phosphorylation negatively modulates tyrosine phosphorylation. Taken together, the results suggest that STAT3 phosphorylation on the serine 727 residue is closely related with TMZ-resistance in TR cells.

STAT3 is transactivated by either phosphorylated or unphosphorylated STAT3 (U-STAT3 or total STAT3) $(37,38)$. U-STAT3 accumulates in the nucleus by binding to unphosphorylated $\mathrm{NF}-\kappa \mathrm{B}$, and this complex, as a novel transcription factor, activates (by a novel mechanism distinct from that used by STAT3 dimers) the transcripton of genes including E2F-1, RANTES, IL-6,IL-8 or MRAS. The products of E2F-1regulated genes, including cyclin $D 1$, cyclin $E$, cyclin $B 1$ and $c d c 2$, contribute to the cell cycle transition and inhibit apoptosis. Notably, NF- $\mathrm{KB}$ is constitutively activated in glioma cells (39); consistent with this, the expression of U-STAT3 was found to be increased in TR cells. Therefore, TMZ-resistance observed in the TR glioma cells may be due to the overexpression of U-STAT3. This suggestion agrees with the observation in this study that siRNA-mediated inhibition of STAT3 reversed TMZ-resistance in TR cells. Further molecular studies are therefore required to elucidate the mechanisms regarding the relationship between TMZ-resistance and pSTAT3-Tyr705, pSTAT3-Ser727 and U-STAT3.

In conclusion, STAT3 activation and expression was found to be elevated in TR cells. Moreover, the inhibition of STAT3 expression results in an increased TMZ sensitivity of TR cells. Thus, STAT3 is a potential molecular target for the development of a novel strategy to inhibit TMZ-resistance in gliomas.

\section{Acknowledgements}

This study was supported by a grant from the National R\&D Program for Cancer Control, Ministry for Health, Welfare and Family Affairs, Republic of Korea (0720330).

\section{References}

1. Wen PY and Kesari S: Malignant gliomas in adults. N Engl J Med 359: 492-507, 2008.

2. Stupp R, Mason WP, van den Bent MJ, et al: Radiotherapy plus concomitant and adjuvant temozolomide for glioblastoma. N Engl J Med 352: 987-996, 2005.
3. Yung WK, Prados MD, Yaya-Tur R, et al: Multicenter phase II trial of temozolomide in patients with anaplastic astrocytoma or anaplastic oligoastrocytoma at first relapse. Temodal Brain Tumor Group. J Clin Oncol 17: 2762-2771, 1999.

4. Balmaceda C, Peereboom D, Pannullo S, et al: Multi-institutional phase II study of temozolomide administered twice daily in the treatment of recurrent high-grade gliomas. Cancer 112: 1139-1146, 2008.

5. Chamberlain MC and Johnston S: Salvage chemotherapy with bevacizumab for recurrent alkylator-refractory anaplastic astrocytoma. J Neurooncol 91: 359-367, 2009.

6. Butowski N, Lamborn KR, Lee BL, et al: A North American brain tumor consortium phase II study of poly-ICLC for adult patients with recurrent anaplastic gliomas. J Neurooncol 91: 183-189, 2009.

7. Fabrini MG, Silvano G, Lolli I, et al: A multi-institutional phase II study on second-line Fotemustine chemotherapy in recurrent glioblastoma. J Neurooncol 92: 79-86, 2009.

8. Wen Z, Zhong Z and Darnell JE Jr: Maximal activation of transcription by Stat 1 and Stat 3 requires both tyrosine and serine phosphorylation. Cell 82: 241-250, 1995.

9. Yu CL, Meyer DJ, Campbell GS, et al: Enhanced DNA-binding activity of a Stat3-related protein in cells transformed by the Src oncoprotein. Science 269: 81-83, 1995.

10. Darnell JE Jr, Kerr IM and Stark GR: Jak-STAT pathways and transcriptional activation in response to IFNs and other extracellular signaling proteins. Science 264: 1415-1421, 1994.

11. Yokogami K, Wakisaka S, Avruch J and Reeves SA: Serine phosphorylation and maximal activation of STAT3 during CNTF signaling is mediated by the rapamycin target mTOR Curr Biol 10: 47-50, 2000.

12. Zhang YW, Wang LM, Jove R and Vande Woude GF: Requirement of Stat3 signaling for HGF/SF-Met mediated tumorigenesis. Oncogene 21: 217-226, 2002.

13. Yu H and Jove R: The STATs of cancer - new molecular targets come of age. Nat Rev Cancer 4: 97-105, 2004.

14. Bromberg J: Stat proteins and oncogenesis. J Clin Invest 109: 1139-1142, 2002.

15. Darnell JE Jr: STATs and gene regulation. Science 277: 1630-1635, 1997.

16. Catlett-Falcone R, Landowski TH, Oshiro MM, et al: Constitutive activation of Stat 3 signaling confers resistance to apoptosis in human U266 myeloma cells. Immunity 10: 105-115, 1999.

17. Duan Z, Foster R, Bell DA, et al: Signal transducers and activators of transcription 3 pathway activation in drug-resistant ovarian cancer. Clin Cancer Res 12: 5055-5063, 2006.

18. D'Atri S, Tentori L, Lacal PM, et al: Involvement of the mismatch repair system in temozolomide-induced apoptosis. Mol Pharmacol 54: 334-341, 1998.

19. Margison GP, Povey AC, Kaina B and Santibanez Koref MF: Variability and regulation of O6-alkylguanine-DNA alkyltransferase. Carcinogenesis 24: 625-635, 2003.

20. Choi $\mathrm{CH}$ : $\mathrm{ABC}$ transporters as multidrug resistance mechanisms and the development of chemosensitizers for their reversal. Cancer Cell Int 5: 30, 2005.

21. Stavrovskaya AA: Cellular mechanisms of multidrug resistance of tumor cells. Biochemistry (Mosc) 65: 95-106, 2000.

22. Yamauchi T, Ogawa M and Ueda T: Carmustine-resistant cancer cells are sensitized to temozolomide as a result of enhanced mismatch repair during the development of carmustine resistance. Mol Pharmacol 74: 82-91, 2008.

23. Pegg AE, Dolan ME and Moschel RC: Structure, function, and inhibition of O6-alkylguanine-DNA alkyltransferase. Prog Nucleic Acid Res Mol Biol 51: 167-223, 1995.

24. Real PJ, Sierra A, De Juan A, Segovia JC, Lopez-Vega JM and Fernandez-Luna JL: Resistance to chemotherapy via Stat3dependent overexpression of $\mathrm{Bcl}-2$ in metastatic breast cancer cells. Oncogene 21: 7611-7618, 2002.

25. Duan Z, Ames RY, Ryan M, Hornicek FJ, Mankin H and Seiden MV: CDDO-Me, a synthetic triterpenoid, inhibits expression of IL-6 and Stat3 phosphorylation in multi-drug resistant ovarian cancer cells. Cancer Chemother Pharmacol 63: 681-689, 2009.

26. Kim JH, Lee SC, Ro J, Kang HS, Kim HS and Yoon S: Jnk signaling pathway-mediated regulation of Stat3 activation is linked to the development of doxorubicin resistance in cancer cell lines. Biochem Pharmacol 79: 373-380, 2010.

27. Rebbaa A, Chou PM and Mirkin BL: Factors secreted by human neuroblastoma mediated doxorubicin resistance by activating STAT3 and inhibiting apoptosis. Mol Med 7: 393-400, 2001. 
28. Zhong Z, Wen Z and Darnell JE Jr: Stat3: a STAT family member activated by tyrosine phosphorylation in response to epidermal growth factor and interleukin-6. Science 264: 95-98, 1994.

29. Wen $Z$ and Darnell JE Jr: Mapping of Stat 3 serine phosphorylation to a single residue (727) and evidence that serine phosphorylation has no influence on DNA binding of Stat1 and Stat3. Nucleic Acids Res 25: 2062-2067, 1997.

30. Kovarik P, Stoiber D, Eyers PA, et al: Stress-induced phosphorylation of STAT1 at Ser727 requires p38 mitogen-activated protein kinase whereas IFN-gamma uses a different signaling pathway. Proc Natl Acad Sci USA 96: 13956-13961, 1999.

31. Jain N, Zhang T, Kee WH, Li W and Cao X: Protein kinase C delta associates with and phosphorylates Stat 3 in an interleukin6-dependent manner. J Biol Chem 274: 24392-24400, 1999.

32. Liu H, Ma Y, Cole SM, et al: Serine phosphorylation of STAT3 is essential for Mcl-1 expression and macrophage survival. Blood 102: 344-352, 2003.

33. Hazan-Halevy I, Harris D, Liu Z, et al: STAT3 is constitutively phosphorylated on serine 727 residues, binds DNA, and activates transcription in CLL cells. Blood 115: 2852-2863, 2010.

34. Qin HR, Kim HJ, Kim JY, et al: Activation of signal transducer and activator of transcription 3 through a phosphomimetic serine 727 promotes prostate tumorigenesis independent of tyrosine 705 phosphorylation. Cancer Res 68: 7736-7741, 2008.
35. Shi X, Zhang H, Paddon H, Lee G, Cao X and Pelech S: Phosphorylation of STAT3 serine-727 by cyclin-dependent kinase 1 is critical for nocodazole-induced mitotic arrest. Biochemistry 45: 5857-5867, 2006.

36. Chung J, Uchida E, Grammer TC and Blenis J: STAT3 serine phosphorylation by ERK-dependent and -independent pathways negatively modulates its tyrosine phosphorylation. Mol Cell Biol 17: 6508-6516, 1997.

37. Yang J, Liao X, Agarwal MK, Barnes L, Auron PE and Stark GR: Unphosphorylated STAT3 accumulates in response to IL-6 and activates transcription by binding to NFkappaB. Genes Dev 21: 1396-1408, 2007.

38. Yang J, Chatterjee-Kishore M, Staugaitis SM, et al: Novel roles of unphosphorylated STAT3 in oncogenesis and transcriptional regulation. Cancer Res 65: 939-947, 2005.

39. Raychaudhuri B, Han Y, Lu T and Vogelbaum MA: Aberrant constitutive activation of nuclear factor kappaB in glioblastoma multiforme drives invasive phenotype. J Neurooncol 85: 39-47, 2007. 